\title{
Characterization of Flow Field Structure and Species Composition in a Shear Coaxial Rocket GH2/GO2 Injector: Modeling of Wall Heat Losses
}

\author{
D. J. Lee*, \\ University of Michigan, Ann Arbor, MI 48109 \\ S. Thakur† J. Wright; \\ Streamline Numerics, Inc., Gainesville, FL 32609 \\ M. Ihme§, and W. Shyy \\ University of Michigan, Ann Arbor, MI 48109
}

\begin{abstract}
A LES combustion model for the prediction of wall-heat transfer in rocket engines and confined combustors is developed. The model employs a flamelet-formulation, in which wall heat-loss effects are introduced by including a source term in the unsteady flamelet equations. This source term is modeled as convective heat-loss process, which evolves on a time-scale that is slow compared to the characteristic chemical scales. This time-scale is modeled by employing a Nusselt-number relation. The thermochemical composition of the non-adiabatic flamelet structure is then obtained from the solution of the unsteady flamelet equations, and is parameterized in terms of mixture fraction, temperature, and scalar dissipation rate. The model is applied in LES of a uni-element rocket injector that was experimentally investigated by Pal et al. [Third Int. Workshop on Rocket Comb. Modeling, Paris, France, March, 13-15, 2006]. Comparisons with adiabatic modeling results show that the consideration of wall-heat losses results in a significant reduction in the temperature in the recirculation zone and a $25 \%$ reduction in the $\mathrm{OH}$ mass fraction at the combustor exit.
\end{abstract}

\section{Introduction}

A ccording to a recent report by Goetz \& Monk, ${ }^{1}$ many of the main-engine failures that have been reported Aduring the early design and development phase of the space shuttle could either be fully or partially attributed to issues associated with the heat transfer and engine cooling systems. These failures indicate that the thermal loading and heat transfer rates to the injector face plate, combustion chamber, and rocket skirt remain largely unknown and therefore represent a major source of uncertainty in the design of rocket engines.

Despite the enormous progress in the development of computational simulation methods for the prediction of rocket engines, only recently has the issue of wall-heat transfer modeling in rocket-engines found some attention. Most notable is the coordinated effort between experimental and computational groups to assess current modeling techniques in predicting the wall-heat transfer in a uni-element rocket injector. ${ }^{2}$ In this work, a single-element $\mathrm{GO}_{2} / \mathrm{GH}_{2}$ coaxial rocket injector configuration due to Pal et al. ${ }^{3}$ was modeled using different simulation techniques. Although various modeling approaches (LES, U/RANS), combustion models (finite-rate chemistry, flamelet formulations), computational setups, and grid-arrangements (two and threedimensional computational domains), as well as different turbulence and subgrid-closure models have been

\footnotetext{
*Graduate Student Research Assistant, Aerospace Engineering Department

$\dagger$ Senior Scientist, Streamline Numerics, Inc.

${ }^{\ddagger}$ Senior Scientist, Streamline Numerics, Inc.

$\S$ Assistant Professor, Aerospace Engineering Department

"Clarence L. "Kelly" Johnson Collegiate Professor, Aerospace Engineering Department; Current affiliation: Honk Kong University of Science and Technology
} 
employed, the outcome of this work represents an important step in establishing a benchmark for assessing the predictive capability of currently rocket-engine modeling capabilities. More recently, Sozer et al. ${ }^{4,5}$ performed comprehensive RANS-investigations of uni- and multi-element rocket-injectors to assess effects of chemical-kinetics mechanisms, turbulence models, resolution requirements, and wall-function formulations.

The objective of this work is to develop a flamelet-based combustion model for the prediction of wall-heat transfer in rocket engines. Compared to detailed chemistry formulations, the advantage of the flameletmodel is its representation of the detailed flame-structure in terms of a reduced set of describing variables (namely mixture fraction, scalar dissipation rate, and a progress variable), and the consideration of turbulence/chemistry interaction by utilizing a presumed probability density function (PDF). Therefore, the associated reduction in computational complexity makes this formulation attractive for practical applications. Currently, steady flamelet formulations are most-commonly employed. The steady-state flamelet approach relies on the underlying assumption that the chemical state of a particular flamelet relaxes to the steady-state solution on a sufficiently fast time-scale. However, heat-loss processes that are associated with radiation and convective heat transfer evolve on time-scales that are slow compared to chemical processes in typical combustion applications. To incorporate non-adiabatic processes into the steady-state flamelet formulation, extensions have been proposed. ${ }^{6-9}$ These extensions mainly considered the modeling of radiation processes, which was achieved by introducing a so-called enthalpy defect variable as an additional reaction coordinate. Steady laminar flamelets are hereby generated by reducing the temperature at the boundaries to achieve a required enthalpy defect that is equivalent to a specified heat-loss. A shortcoming of this procedure is that it leads to unrealistic low temperatures at the boundaries for large enthalpy defects. This is remedied by either reducing the solution domain in mixture fraction space $^{7}$ or changing the mixture composition at the boundaries. ${ }^{8}$ Furthermore, the somewhat arbitrary specification of a uniform enthalpy defect across a laminar flamelet may result in an unphysical description of the corresponding flame structure. Nevertheless, it was demonstrated in different applications that these models lead to improved temperature predictions.

While the concept of a defect-enthalpy can be extended to predict convective heat-loss processes for moderate heat-transfer ${ }^{10}$ several challenges arise for combustor applications that are characterized by significant heat-losses. In particular, strong heat-loss can lead to flame-quenching, and the change in enthalpy during the quenching process cannot be uniquely represented in terms of a defect enthalpy. This aspect will be further discussed in the context of Fig. 5. Furthermore, radiative heat losses are commonly represented in the optical thin-limit, ${ }^{11}$ resulting in a point-local source term representation. However, the heat flux is a vectorial quantity, and is typically not aligned with the one-dimensional flamelet structure. In the context of the flamelet-model it is therefore more suitable to represent wall-heat losses in terms of a convective heattransfer. This is done in the following work, and a time-scale is identified that is associated with the slow convective heat-loss process. This time-scale is subsequently modeled in terms of a Nusselt-number relation.

The significance of radiative and convective heat-loss contributions can be estimated by considering the following dimensionless quantities ${ }^{\mathrm{a}}$ :

$$
\begin{aligned}
& \mathcal{R}=\frac{\text { Radiative Heat Loss }}{\text { Enthalpy }}=\frac{4 \sigma T_{\text {ref }}^{3}}{\rho_{\text {ref }} c_{p, \text { ref }} U_{\text {ref }}}, \\
& \mathcal{C}=\frac{\text { Convective Heat Loss }}{\text { Enthalpy }}=\frac{\mathrm{Nu}}{\operatorname{Pr} \operatorname{Re}},
\end{aligned}
$$

where $\sigma$ is the Steffan-Boltzmann constant, $T$ is the temperature, $\rho$ the density, $c_{p}$ the specific heat, $U$ the velocity, $D$ the diameter, and the subscript "ref" denotes a reference quantity. Considering a stoichiometric hydrogen/oxygen combustion at conditions specified in Sec. III, values for these quantities can be estimated as $\mathcal{R} \approx 1.2 \times 10^{-3}$ and $\mathcal{C} \approx 3.1 \times 10^{-3}$. This estimate indicates that convective heat-losses are approximately three times larger compared to radiative heat-losses. Therefore, radiation effects are not considered in the present work, and the main focus is on the modeling of wall-heat losses. The mathematical model is described in the next section. This non-adiabatic flamelet-model is the applied in LES of a uni-element rocket injector. The experimental configuration is presented in Sec. III, and results are discussed in Sec. IV. The paper finishes with conclusions.

\footnotetext{
${ }^{a}$ The following values were used for computing the non-dimensional quantities $\mathcal{R}$ and $\mathcal{C}: \sigma=5.67 \times 10^{-8} \mathrm{~W} /\left(\mathrm{m}^{2} \mathrm{~K}^{2}\right)$, $T_{\text {ref }}=3500 \mathrm{~K}, U_{\text {ref }}=740 \mathrm{~m} / \mathrm{s}, D_{\text {ref }}=7.49 \mathrm{~mm}, \rho_{\text {ref }}=3.456 \mathrm{~kg} / \mathrm{m}^{3}, c_{p, \text { ref }}=3.16 \mathrm{~kJ} /(\mathrm{kg} \mathrm{K}), \nu_{\mathrm{ref}}=7.055 \times 10^{-6} \mathrm{~m}^{2} / \mathrm{s}$, $\lambda_{\text {ref }}=0.476 \mathrm{~W} /(\mathrm{m} \mathrm{K}), \mathrm{Pr}=0.162, \mathrm{Re}=785,600$, and $\mathrm{Nu}=400$.
} 


\section{Mathematical Model}

\section{A. Governing Equations}

The instantaneous conservation equations for mass, momentum, mixture fraction, species mass fraction, and temperature can be written in dimensionless form as:

$$
\begin{aligned}
\mathcal{D}_{t} \rho & =-\rho \nabla \cdot \boldsymbol{u} \\
\rho \mathcal{D}_{t} \boldsymbol{u} & =-\nabla p+\frac{1}{\operatorname{Re}} \nabla \cdot \underline{\underline{\tau}}, \\
\rho \mathcal{D}_{t} Z & =\frac{1}{\operatorname{ReSc}} \nabla \cdot(\rho \alpha \nabla Z) \\
\rho \mathcal{D}_{t} \boldsymbol{Y} & =\frac{1}{\operatorname{ReSc}} \nabla \cdot(\rho \alpha \nabla \boldsymbol{Y})+\dot{\boldsymbol{m}} \\
\rho c_{p} \mathcal{D}_{t} T & =\frac{1}{\operatorname{ReSc}} \nabla \cdot(\lambda \nabla T)+\dot{Q}_{H}-\frac{1}{\operatorname{ReSc}}\left(\rho \sum_{i} c_{p, i} Y_{i} \boldsymbol{v}_{i}\right) \cdot \nabla T+\frac{\mathrm{Ec}}{\operatorname{Re}} \underline{\underline{\tau}}: \nabla \boldsymbol{u}+\mathrm{Ec} \mathcal{D}_{t} p+\mathcal{R} \dot{Q}_{R},
\end{aligned}
$$

in which $\boldsymbol{u}$ is the velocity vector, $p$ is the pressure, $\underline{\underline{\tau}}$ is the viscous stress tensor, and $\mathcal{D}_{t}=\partial_{t}+\boldsymbol{u} \cdot \nabla$ is the substantial derivative. The mixture fraction is denoted by $Z, \alpha$ is the species diffusivity, $T$ is the temperature, $c_{p}$ is the specific heat at constant pressure, $\lambda$ is the heat conductivity, $\boldsymbol{v}_{k}$ is the diffusion velocity of species $i, \dot{Q}_{H}$ is the heat release rate and $\dot{Q}_{R}=4 \sigma\left(T_{\infty}^{4}-T^{4}\right) \sum_{i} p_{i} a_{i}$ is the radiative heat loss, which is here represented in the optical thin limit. ${ }^{12,13}$ The following dimensionless variables are used in Eqs. (2):

$$
\begin{aligned}
& \mathcal{D}_{t}=\frac{D_{\text {ref }}}{U_{\text {ref }}} \mathcal{D}_{t}^{*}, \quad \nabla=D_{\text {ref }} \nabla^{*}, \quad \boldsymbol{u}=\frac{\boldsymbol{u}^{*}}{U_{\text {ref }}}, \quad \rho=\frac{\rho^{*}}{\rho_{\text {ref }}}, \quad p=\frac{p^{*}}{\rho_{\text {ref }} U_{\text {ref }}^{2}}, \\
& \nu=\frac{\nu^{*}}{\nu_{\mathrm{ref}}}, \quad \alpha=\frac{\alpha^{*}}{\alpha_{\mathrm{ref}}}, \quad \lambda=\frac{\lambda^{*}}{\lambda_{\mathrm{ref}}}, \quad c_{p}=\frac{c_{p}^{*}}{c_{p, \mathrm{ref}}}, \quad \dot{Q}_{H}=\frac{\dot{Q}_{H}^{*} D_{\mathrm{ref}}}{U_{\mathrm{ref}} \rho_{\mathrm{ref}} c_{p, \mathrm{ref}} T_{\mathrm{ref}}}, \\
& \dot{\boldsymbol{m}}=\frac{\dot{\boldsymbol{m}}^{*} D_{\mathrm{ref}}}{U_{\mathrm{ref}} \rho_{\mathrm{ref}}}, \quad \boldsymbol{v}=\frac{\boldsymbol{v} D_{\mathrm{ref}}}{\alpha_{\mathrm{ref}}}, \quad T=\frac{T^{*}}{T_{\mathrm{ref}}}, \quad \underline{\underline{\tau}}=\frac{\underline{\underline{\tau}}^{*} D_{\mathrm{ref}}}{\rho_{\mathrm{ref}} \nu_{\mathrm{ref}} U_{\mathrm{ref}}}, \quad \dot{Q}_{R}=\frac{\dot{Q}_{R}^{*} D_{\mathrm{ref}}}{4 \sigma T_{\mathrm{ref}}^{4}},
\end{aligned}
$$

and the asterisk refers to a dimensional quantity. Using this non-dimensionalization together with the heatloss parameters defined in Eqs. (1), the following relevant similarity parameters can be identified:

$$
\begin{aligned}
\text { Reynolds number: } & \mathrm{Re}=\frac{U_{\text {ref }} D_{\text {ref }}}{\nu_{\text {ref }}}, \\
\text { Schmidt number: } & \mathrm{Sc}=\frac{\nu_{\text {ref }}}{\alpha_{\text {ref }}}, \\
\text { Eckert number: } & \mathrm{Ec}=\frac{U_{\text {ref }}^{2}}{c_{\mathrm{p}, \mathrm{ref}} T_{\text {ref }}}, \\
\text { Lewis number: } & \mathrm{Le}=\frac{\lambda_{\text {ref }}}{\rho_{\text {ref }} c_{\mathrm{p}, \mathrm{ref}} \alpha_{\text {ref }}}, \\
\text { Prandtl number: } & \operatorname{Pr}=\frac{c_{\mathrm{p}, \mathrm{ref}} \rho_{\text {ref }} \nu_{\text {ref }}}{\lambda_{\text {ref }}}=\frac{\mathrm{Sc}}{\mathrm{Le}} .
\end{aligned}
$$

\section{B. Flamelet Formulation}

In the laminar flamelet model, a turbulent diffusion flame is considered as an ensemble of laminar flamelets. ${ }^{14,15}$ At sufficiently large Damköhler number or sufficiently high activation energy, chemical reactions and heat transfer occur in a thin layer. If the characteristic length scale of this layer is smaller than that of the surrounding turbulence, the turbulent structures cannot penetrate the reaction zone and are unable to destroy the flame structure. The effect of turbulence in this so-called flamelet regime results in a deformation and stretching of the flame sheet. With this notion, a flamelet can be considered as a thin reaction zone surrounded by a molecular transport layer, which, in turn, is embedded within a turbulent flow. ${ }^{16}$

The one-dimensional laminar flamelet equations can be derived by introducing a new coordinate system that is locally attached to the surface of stoichiometric mixture. If the direction normal to the flame surface is 
associated with the mixture fraction, and spatial changes along the other coordinate directions are neglected, the following one-dimensional flamelet equations can be derived for unity-Lewis number flame-regimes: ${ }^{17}$

$$
\begin{aligned}
\rho \partial_{t} \boldsymbol{Y}-\rho \frac{\chi_{Z}}{2} \frac{\partial^{2} \boldsymbol{Y}}{\partial Z^{2}} & =\dot{\boldsymbol{m}}, \\
\rho \partial_{t} T-\rho \frac{\chi_{Z}}{2} \frac{\partial^{2} T}{\partial Z^{2}}-\rho \frac{\chi_{Z}}{2} \frac{1}{c_{p}} \frac{\partial c_{p}}{\partial Z} \frac{\partial T}{\partial Z} & =\frac{\dot{Q}_{H}}{c_{p}},
\end{aligned}
$$

and the scalar dissipation rate is defined as

$$
\chi_{Z}=2|\alpha \nabla Z|^{2}
$$

Under the assumption that all species are formed on time scales that are short compared to those associated with hydrodynamic and heatloss effects, the temporal derivatives in Eqs. (4) are neglect. The resulting steady-state flamelet equations are a more convenient form for practical applications as they are time-independent so that all thermochemical species can then be represented only in terms of the scalar dissipation rate and mixture fraction. For application to LES and RANS, the solution of the flamelet equations is precomputed for a prescribed range of scalar dissipation rates and prescribed boundary conditions in the oxidizer $(Z=0)$ and fuel $(Z=1)$ streams, respectively.

The solution to the steady flamelet equations

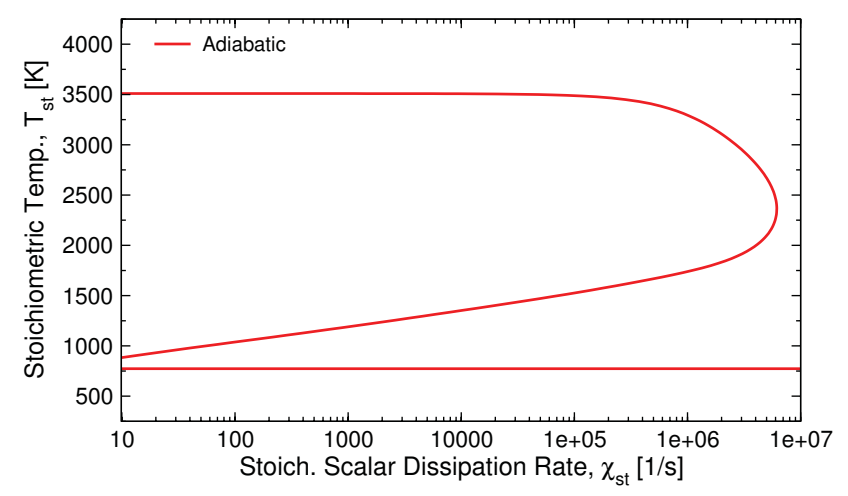

Figure 1. Solution of the steady flamelet equations showing temperature as function of scalar dissipation rate at stoichiometric condition for a $\mathrm{H}_{2} / \mathrm{O}_{2}$ flame; composition equal to that specified in Tab. 1 .

is illustrated in Fig. 1, showing temperature as function of scalar dissipation rate at stoichiometric condition. In this figure, three distinct branches can be identified, corresponding to the upper stable branch which is associated with the stable burning flame, the middle unstable branch, and the lower branch which corresponds to the mixing between reactants.

\section{Modeling of Heat-Loss Effects in Flamelet Formulation}

In the following, we are concerned with the modeling of wall-heat loss effects in the context of the laminar flamelet formulation. While effects of thermal radiation have been previously considered in the flamelet formulation, heat-loss effects by forced convection and conduction are difficult to incorporate into Eqs. (4). Reason for this is the fact that these heat losses are localized in physical space, and can therefore not directly be represented in the flamelet equations, which are solved in mixture-fraction space. To illustrate this, consider Fig. 2 showing two different scenarios of convective heat transfer from the flame to the combustor walls, having a constant temperature $T_{\text {wall }}$.

Figure 2(a) illustrates the situation in which the heat flux is primarily aligned with the mixture-fraction gradient. This heat-transfer scenario can be incorporated into the flamelet equations by solving Eqs. (4) in the interval $\left[0, Z\left(t, \boldsymbol{x}_{\text {wall }}\right)\right]$ or $\left[Z\left(t, \boldsymbol{x}_{\text {wall }}\right), 1\right]$, dependent on whether the fuel-lean or fuel rich-side is aligned with the wall, which is here denoted by $\boldsymbol{x}_{\text {wall }}$. Appropriate boundary conditions for species composition, temperature, and scalar-dissipation rate are then enforced in the 'boundary stream' $Z_{\text {wall }}=Z\left(t, \boldsymbol{x}_{\text {wall }}\right)$. However, due to the transient flame evolution inside the combustor, the value of $Z_{\text {wall }}$ is dependent on time and space, so that this approach requires a time-dependent solution approach. This, in turn, introduces additional challenges for engineering applications for the reason that the flamelet solution is transient and conventional tabulation approaches cannot be employed.

Wall-heat transfer perpendicular to the mixture-fraction gradient is shown in Fig. 2(b). Under this condition, the entire flame-structure is affected by heat losses. Since the flamelet-formulation is a onedimensional representation of a diffusion flame structure, this wall-heat transfer scenario cannot be directly represented in terms of a conductive heat flux term. 


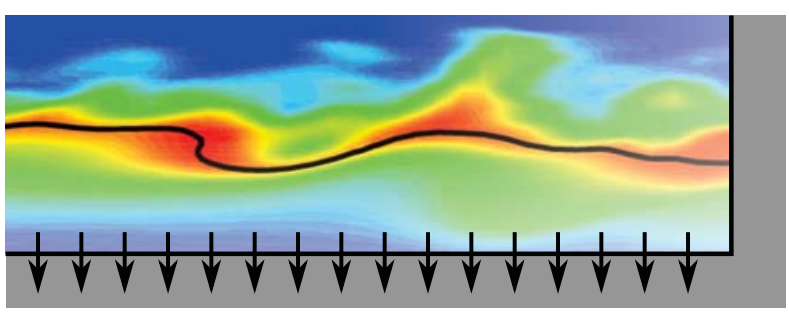

(a) Flame-normal wall heat transfer.

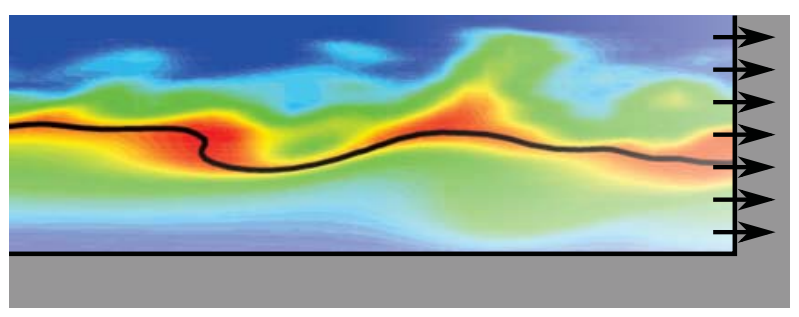

(b) Flame-parallel wall heat transfer.

Figure 2. Schematic of two wall-heat-loss scenarios: (a) flame-normal heat transfer in which the heat-flux vector is aligned with the mixture-fraction gradient, and (b) flame-parallel heat transfer in which mixture-fraction gradient and heat-flux vector are misaligned.

In the following, heat-loss effects are modeled in terms of forced convective heat transfer. To this end, the following convective heat-loss term is added to the right-hand-side of the temperature equation, Eq. (4b):

$$
\dot{Q}_{C}=\frac{1}{\tau_{C}} \rho\left(T_{\text {wall }}-T\right) .
$$

The convective time-scale $\tau_{C}$ can be expressed in terms of heat transfer coefficient $h$, specific heat capacity, density, and a length-scale $\Delta$ :

$$
\tau_{C}=\frac{c_{p} \rho}{h} \Delta .
$$

The heat transfer coefficient can be related to the heat conductivity via a Nusselt number relation,

$$
\mathrm{Nu}=\frac{D_{\mathrm{ref}} h}{\lambda}
$$

which compares convective and conductive heat transfer rates. Although Nusselt-number relations for reacting flows are not reported, in the following we assume that the flow in the near-wall region of the rocketchamber is fully-developed and turbulent. With this assumption, we approximate the Nusselt number by the following correlation function: ${ }^{18}$

$$
\mathrm{Nu}=\frac{\xi \operatorname{Re} \operatorname{Pr}}{k+12.7 \sqrt{\xi}\left(\operatorname{Pr}^{2 / 3}-1\right)},
$$

which has been established for fully-developed turbulent flows in cylindrical pipes. The parameters $\xi$ and $k$ in Eq. (9) are functions of Reynolds and Prandtl numbers, and are given as

$$
\begin{aligned}
k & =1.07+\frac{900}{\operatorname{Re}}-\frac{0.63}{1+10 \operatorname{Pr}}, \\
\xi & =\frac{1}{8}\left[1.82 \log _{10}(\mathrm{Re})-1.64\right]^{-2} .
\end{aligned}
$$

The Nusselt-number relation (9) is illustrated in Fig. 3 as function of Reynolds-number for different values of Pr.

In this context it is pointed out that the herein proposed non-adiabatic flamelet model is not particularly sensitive to the Nusselt-number relation. This is due to the fact that the convective heat loss is a slow process and evolves on a time-scale that is long compared to the characteristic chemical time-scale, $\tau_{\text {chem }}$. This can be illustrated by the ratio

$$
\begin{aligned}
\frac{\tau_{C}}{\tau_{\text {chem }}} & =\frac{\tau_{C}}{\tau_{\text {flow }}} \frac{\tau_{\text {flow }}}{\tau_{\text {chem }}}, \\
& =\frac{1}{\mathcal{C}} \mathrm{Da}
\end{aligned}
$$

with $\mathcal{C}$ from Eq. (1b) and Da is the Damköhler number. This relation shows that for large Damköhler-number flame regimes the convective time-scale is several orders of magnitude smaller than the chemical time-scale, 


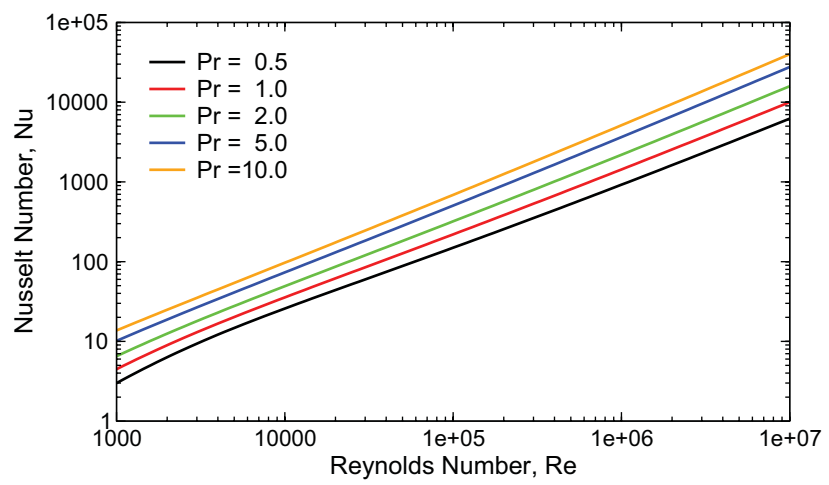

Figure 3. Nusselt-number, evaluated from Eq. (9) as function of Reynolds-number and different values of Prandtl number.

so that the flame-structure reaches a steady state and the chemistry is not affected by transient effects. As such, the convective time-scale only controls the heat-loss rate.

Effects of wall heat losses on the flamelet solution are illustrated in Fig. 4, showing a comparison between the adiabatic S-shaped curve (red) and the solution of the steady flamelet equations under consideration of convective heat losses (blue dashed line). The fuel/air composition is identical to that specified in Tab. 1, and the wall temperature is $T_{\text {wall }}=500 \mathrm{~K}$. From this figure, it can be seen that heat losses mainly affect the flame structure at low scalar dissipation rates and become increasingly insignificant for larger dissipation rates. Note also that heat losses result in a decrease in the quenching dissipation rate $\chi_{\mathrm{st}, \mathrm{q}}$. Flamelets that are obtained from the solution of the unsteady flamelet equations are shown by black symbols. These unsteady flamelet calculations are initialized with the steady-state adiabatic flamelet solution and integrated for a fixed scalar dissipation rate until the steady non-adiabatic flame-state is reached.

In the following, the transient flamelet solution (shown by the black symbols in Fig. 4) is parameterized in terms of mixture fraction, temperature, and stoichiometric scalar dissipation rate, viz.,

$$
\phi=\phi\left(Z, T, \chi_{Z, \mathrm{st}}\right)
$$

where $\phi=\left(\boldsymbol{Y}, \dot{\boldsymbol{m}}, \dot{Q}_{H}, \rho, \nu, \alpha, c_{p}\right)^{T}$, and $\chi_{Z, \mathrm{st}}=\chi_{Z} F(Z)^{-1}$ with $^{17}$

$$
F(Z)=\exp \left\{2\left(\left[\operatorname{erfc}^{-1}\left(2 Z_{\mathrm{st}}\right)\right]^{2}-\left[\operatorname{erfc}^{-1}(2 \mathrm{Z})\right]^{2}\right)\right\}
$$

In this context it is noted that the total enthalpy is commonly used for the parameterization of nonadiabatic systems. ${ }^{6-10,19}$ However, for the case of flame-quenching and variations in the temperature boundary conditions, the enthalpy is a non-monotonic function. This is illustrated in Fig. 5, showing (a) the

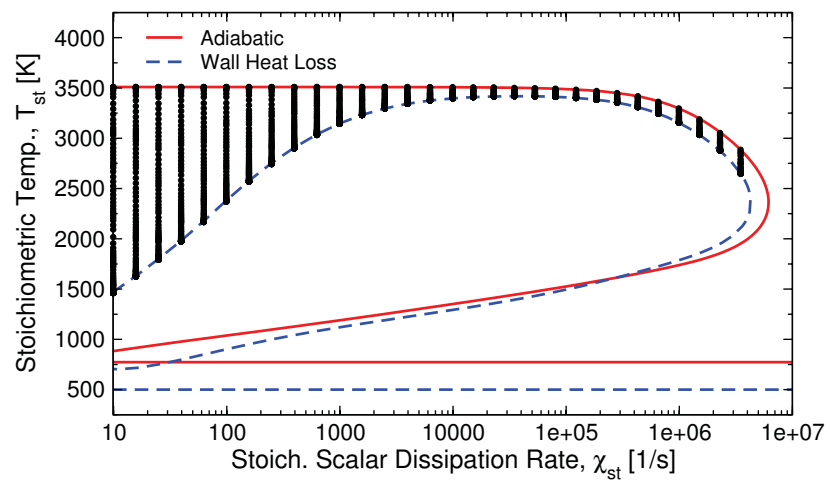

Figure 4. Solution of the steady flamelet equations showing temperature as function of scalar dissipation rate at stoichiometric condition for a $\mathrm{H}_{2} / \mathrm{O}_{2}$ flame; red line shows the adiabatic steady-state solution; blue dashed line corresponds to the steady-state flamelet-solution under consideration of wall-heat losses, and black symbols denote unsteady flamelet-solutions; composition is equal to that specified in Tab. 1 and wall temperature is $500 \mathrm{~K}$. 


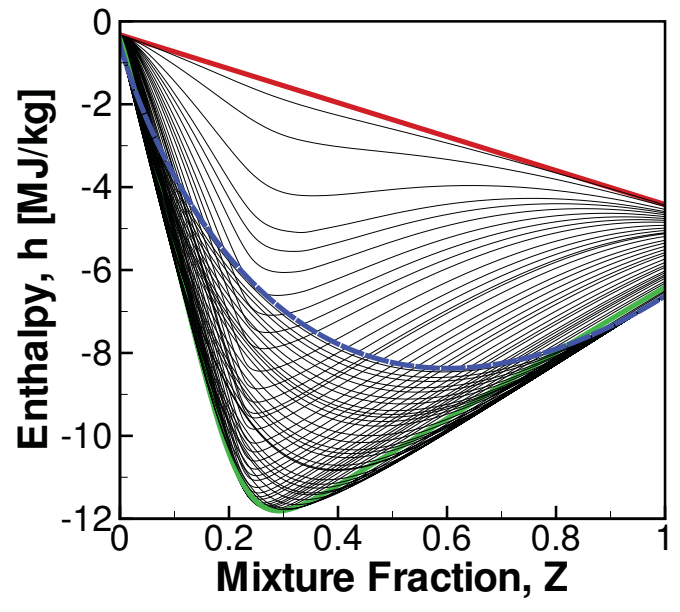

(a)

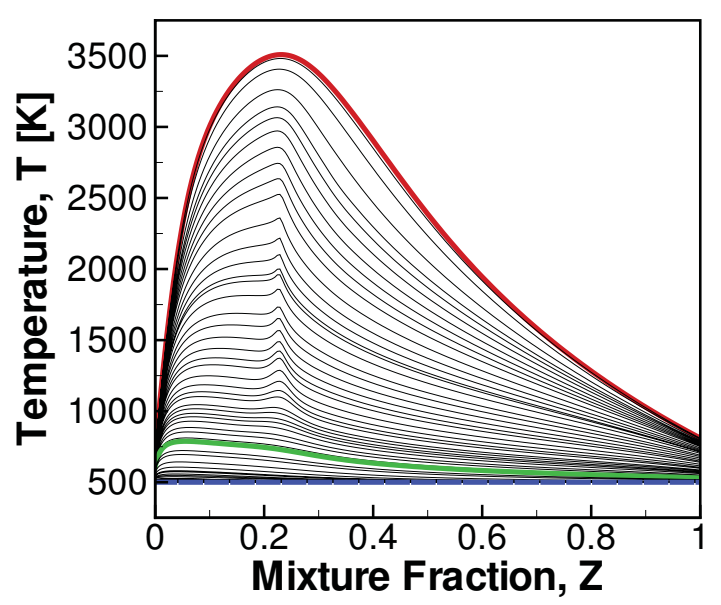

(b)

Figure 5. Evolution of (a) total enthalpy (consisting of chemical and sensible contributions) and (b) temperature for a constant scalar dissipation rate of $\chi_{Z, s t}=0.1 \mathbf{s}^{-1}$. The red line corresponds to the adiabatic solution and the blue dashed line denotes the steady solution for the case with wall-heat losses.

temporal evolution of the total enthalpy and (b) the corresponding flamelet temperature for a fixed scalar dissipation rate of $\chi_{Z \text {,st }}=0.1 \mathrm{~s}^{-1}$. This figure shows that the temperature is monotonic, and is therefore used for the parameterization of the flamelet solutions.

\section{LES Combustion Model}

In LES, the coherent large scale structures of the turbulent flow are computationally resolved, and effects of the smaller and numerically unresolved scales on the large scales are modeled. The decomposition of the scales is achieved by applying a low-pass filter to the flow field quantities, and - in the case of a reacting flow - the Favre-filtered quantity of a scalar $\psi$ is computed as:

$$
\widetilde{\psi}(t, \boldsymbol{x})=\frac{1}{\bar{\rho}} \int \rho(t, \boldsymbol{x}) \psi(t, \boldsymbol{x}) G(t, \boldsymbol{x}, \boldsymbol{y} ; \Delta) d \boldsymbol{y},
$$

where $\Delta$ denotes the filter size, which corresponds in the case of an implicit LES to the local LES grid size. The residual field is defined as $\psi^{\prime \prime}(t, \boldsymbol{x})=\psi(t, \boldsymbol{x})-\widetilde{\psi}(t, \boldsymbol{x})$, and Favre-filtered quantities are related to Reynolds-filtered quantities by $\bar{\rho} \widetilde{\psi}=\overline{\rho \psi}$.

After applying the filter operator to Eqs. (2), the Favre-filtered equations can be written as

$$
\begin{aligned}
\widetilde{\mathcal{D}}_{t} \bar{\rho}= & -\bar{\rho} \nabla \cdot \widetilde{\boldsymbol{u}} \\
\bar{\rho} \widetilde{\mathcal{D}}_{t} \widetilde{\boldsymbol{u}}= & -\nabla \bar{p}+\frac{1}{\operatorname{Re}} \nabla \cdot \underline{\underline{\underline{\tau}}}-\nabla \cdot \underline{\underline{\tau}}^{\mathrm{res}}, \\
\bar{\rho} \widetilde{\mathcal{D}}_{t} \widetilde{Z}= & \frac{1}{\operatorname{ReSc}} \nabla \cdot(\bar{\rho} \widetilde{\alpha} \nabla \widetilde{Z})-\nabla \cdot \boldsymbol{\tau}_{Z}^{\mathrm{res}}, \\
\bar{\rho} \widetilde{\mathcal{D}}_{t} \widetilde{T}= & \frac{1}{\operatorname{ReSc}} \nabla \cdot(\bar{\rho} \widetilde{\alpha} \nabla \widetilde{T})-\nabla \cdot \boldsymbol{\tau}_{T}^{\mathrm{res}}+{\overline{\dot{Q}_{H}} / c_{p}}+ \\
& \frac{1}{\operatorname{ReSc}} \bar{\rho} \widetilde{\alpha}\left[\widetilde{\partial_{Z} \ln \left(c_{p}\right)}+\sum_{i} \widetilde{c}_{p, i} \widetilde{\partial_{Z} Y_{i}}\right] \nabla \widetilde{Z} \cdot \nabla \widetilde{T} .
\end{aligned}
$$

The residual stress tensor $\underline{\underline{\tau}}^{\text {res }}=\bar{\rho} \widetilde{\boldsymbol{u} u}-\bar{\rho} \widetilde{\boldsymbol{u}} \widetilde{\boldsymbol{u}}$ and turbulent fluxes $\boldsymbol{\tau}_{\psi}^{\text {res }}=\bar{\rho} \widetilde{\boldsymbol{u} \psi}-\bar{\rho} \widetilde{\boldsymbol{u}} \widetilde{\psi}$ are modeled by a dynamic Smagorinsky model. ${ }^{20,21}$ The term in square brackets in Eq. (15d) has been derived by expanding the thermochemical quantities and species mass fractions to first-order in terms of mixture fraction, neglecting 
variations in temperature and scalar-dissipation rate. Analysis in the context of a laminar diffusion flame demonstrated the accuracy of this approximation.

The state relation, Eq. (12), is expressed in terms of Favre-averaged quantities by employing a presumed PDF-closure. To this end, Eq. (12) is integrated over a presumed joint probability density function (PDF) for mixture fraction, temperature, and stoichiometric scalar dissipation rate:

$$
\widetilde{\phi}=\iiint \phi\left(Z, T, \chi_{Z, \mathrm{st}}\right) \widetilde{P}\left(Z, T, \chi_{Z, \mathrm{st}}\right) d Z d T d \chi_{Z, \mathrm{st}},
$$

and $\widetilde{P}\left(Z, T, \chi_{Z, \mathrm{st}}\right)$ denotes the density-weighted joint PDF with

$$
\widetilde{P}\left(Z, T, \chi_{Z, \mathrm{st}}\right)=\frac{\rho}{\bar{\rho}} P\left(Z, T, \chi_{Z, \mathrm{st}}\right)=\widetilde{P}\left(Z, \chi_{Z, \mathrm{st}}\right) P\left(T \mid Z, \chi_{Z, \mathrm{st}}\right) .
$$

In the following, this PDF is modeled as:

$$
\widetilde{P}\left(Z, T, \chi_{Z, \mathrm{st}}\right)=\beta\left(Z ; \widetilde{Z}, \widetilde{Z^{\prime \prime 2}}\right) \delta(\widetilde{T}-T \mid Z) \delta\left(\widetilde{\chi} Z, \mathrm{st}-\chi_{Z, \mathrm{st}}\right),
$$

where a beta-PDF is used to model the mixture fraction distribution, and the distributions of $\chi_{Z \text {,st }}$ and $T$ are modeled by a delta function. After computing the Favre-averaged thermochemical quantities, the flameletlibrary which is used for the LES-calculations can then be parameterized in terms of the mean and variance of mixture fraction, mean temperature, and mean stoichiometric scalar-dissipation rate, respectively. This expression can be written in the following form:

$$
\widetilde{\phi}=\widetilde{\phi}\left(\widetilde{Z}, \widetilde{Z^{\prime \prime 2}}, \widetilde{T}, \widetilde{\chi}_{Z, \mathrm{st}}\right)
$$

This formulations shares similarities with the flamelet/progress variable (FPV) formulation, ${ }^{22,23}$ in which the reaction progress variable $C$ is replaced by the temperature $T$.

\section{Experimental Configuration and Computational Setup}

The experimental configuration that is considered in this work consists of the uni-element rocket injector configuration that was experimentally investigated by Pal et al. ${ }^{3}$ The experimental configuration consists of the main rocket chamber having a diameter of $38.1 \mathrm{~mm}$. Two propellant preburners supply gaseous hydrogen and oxygen to the coaxial injector. The central injector nozzle has a diameter of $5.26 \mathrm{~mm}$ and supplies the oxidizer having a composition of $Y_{\mathrm{O}_{2}}=0.9458$ and $Y_{\mathrm{H}_{2} \mathrm{O}}=0.0542$ at a temperature of $T=700 \mathrm{~K}$. The fuel is supplied by an annulus surrounding the inner oxidizer stream, having inner and outer diameters of $6.3 \mathrm{~mm}$ and $7.49 \mathrm{~mm}$ ( $\left.\equiv D_{\text {ref }}\right)$, re-

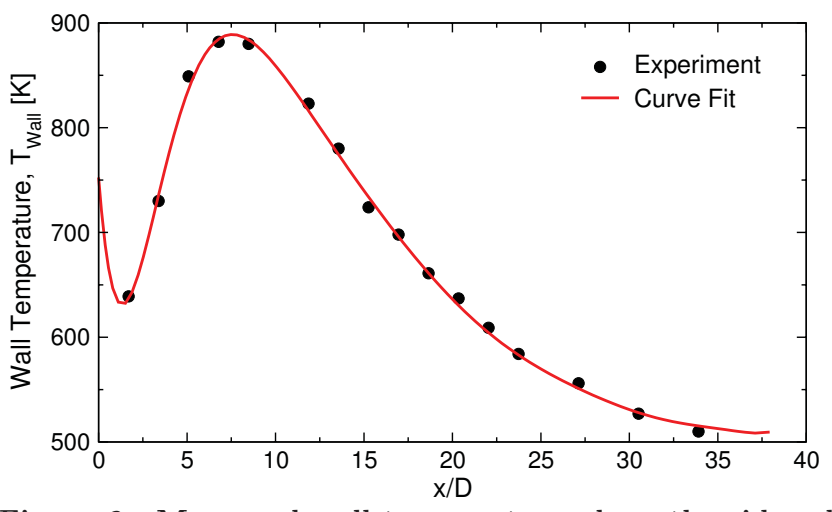

Figure 6. Measured wall temperature along the sidewall of the rocket chamber.

spectively. The fuel composition is $Y_{\mathrm{H}_{2}}=0.4018$ and $Y_{\mathrm{H}_{2} \mathrm{O}}=0.5982$ and the temperature is $T=811 \mathrm{~K}$. The stoichiometric mixture fraction is $Z_{\mathrm{st}}=0.2273$. The post, separating the fuel and oxidizer stream, is recessed with respect to the injector end wall. The post-recession affects the mixing, and was estimated to be 0.43 $\mathrm{mm}$ at fired condition. The pressure in the chamber during the steady-state operation was reported to be 54.2 bar. Although no species composition was recorded during operation, thermocouples were mounted to the chamber wall to measure wall temperature and heat flux. The wall temperature measurements that were used as boundary conditions in the simulation are shown in Fig. 6. For reference, all operating conditions are summarized in Tab. 1.

The Favre-filtered governing equations are solved in cylindrical coordinates. ${ }^{22}$ The geometry is nondimensionalized by the jet nozzle diameter $D_{\text {ref }}$. The computational domain in axial direction extends up to 26 diameters. The axial direction is discretized with 256 grid points following a linear growth rate, and 200 grid points are used in radial direction. The circumferential direction is equally spaced and uses 64 points, resulting in a total number of approximately 3.3 million grid points. 


\begin{tabular}{|c||c|c|}
\hline & Fuel-Stream & Oxidizer-Stream \\
\hline \hline Mixture & $Y_{\mathrm{H}_{2}}=0.4018$ & $Y_{\mathrm{O}_{2}}=0.9458$ \\
& $Y_{\mathrm{H}_{2} \mathrm{O}}=0.5982$ & $Y_{\mathrm{H}_{2} \mathrm{O}}=0.0542$ \\
Stoich. Mixture, $Z_{\mathrm{st}}$ & \multicolumn{2}{|c}{0.2273} \\
Pressure [bar] & \multicolumn{2}{|c}{54.2} \\
Temperature [K] & 811 & 700 \\
Density $\left[\mathrm{kg} / \mathrm{m}^{3}\right]$ & 3.456 & 28.58 \\
Velocity $[\mathrm{m} / \mathrm{s}]$ & $740\left(\equiv U_{\text {ref }}\right)$ & 146 \\
Reynolds Number, Re & 785,600 & 588,000 \\
\hline
\end{tabular}

Table 1. Operating conditions and mixture composition of uni-element rocket-injector.

The turbulent inflow velocity profiles for the fuel and oxidizer streams were generated from a periodic pipe flow simulation. The flamelet calculations were performed using the FlameMASTER code, ${ }^{24}$ and the $\mathrm{H}_{2} / \mathrm{O}_{2}$-chemistry was described by the detailed mechanism due to O'Conaire et al. ${ }^{25}$

\section{Results}

In the following, LES-results will be presented and different modeling assumptions will be investigated. To this end, several computations are performed in order to assess effects of finite-rate chemistry and wallheat losses on the flow-field structure. For reference, the model formulations considered are summarized in Tab. 2.

\begin{tabular}{|c|c|c|}
\hline Model & Formulation, Eq. (19) & Comments \\
\hline \hline $\operatorname{FPV}_{\{\text {Equ, Adiab }\}}$ & $\widetilde{\phi}\left(\widetilde{Z}, \widetilde{Z^{\prime \prime 2}} \mid \widetilde{\chi} Z\right.$,st $\left.=10 \mathrm{~s}^{-1}\right)$ & Equilibrium Chemistry, Adiabatic \\
$\mathrm{FPV}_{\{\text {Adiab }\}}$ & $\widetilde{\phi}\left(\widetilde{Z}, \widetilde{Z^{\prime \prime 2}}, \widetilde{\chi} Z\right.$,st $)$ & Adiabatic, upper stable branch of S-shape curve \\
$\mathrm{FPV}_{\{\text {Non-Adiab }\}}$ & $\widetilde{\phi}\left(\widetilde{Z}, \widetilde{Z}^{\prime \prime 2}, \widetilde{T}, \widetilde{\chi} Z\right.$,st $)$ & Non-adiabatic model with convective heat-loss from Eq. (6) \\
\hline
\end{tabular}

Table 2. Model formulations and simplifications.

\section{A. Non-Equilibrium Chemistry Effects}

The hydrogen/oxygen chemistry is highly reactive and - over a wide range - rather insensitive to strain-rate effects. This is illustrated by the S-shaped curve in Fig. 1, having a quenching value of $\chi_{\mathrm{st}, \mathrm{q}}=6 \times 10^{6} \mathrm{~s}^{-1}$, which is several orders of magnitude larger compared to $\mathrm{CH}_{4} / \mathrm{O}_{2}$-systems and other hydrocarbon fuels. From this figure it can also be deduced that for $\chi_{\mathrm{st}} \leq 10^{5} \mathrm{~s}^{-1}$ flame-strain has a negligible effect on the flamestructure. To investigate the role of non-equilibrium effects in the context of this uni-element rocket injector, two LES-computations are performed. To isolate contributions arising from the wall heat transfer, we hereby considered the adiabatic FPV-formulation. In the first calculation, designated as "FPV $\{$ Equ, Adiab $\}$," the chemistry is described from an equilibrium flamelet solution, which is computed for a stoichiometric scalar dissipation rate of $\chi_{\mathrm{st}}=10 \mathrm{~s}^{-1}$. The second calculation, $\mathrm{FPV}_{\{\text {Adiab }\}}$, uses the flamelet-solution along the entire S-shape curve to describe the thermochemical quantities.

Comparison of temperature fields and radial profiles obtained from both models are illustrated in Figs. 7 and 8. It can be seen that both models give qualitatively and quantitatively similar results. This is particularly evident from the comparison of the radial profiles (Fig. 8), showing virtually identical temperature profiles. For further investigation, the scalar dissipation rate near the injector is evaluated. The mean and instantaneous results are illustrated in Fig. 9. This figure shows that the maximum instantaneous dissipation rate does not exceed values of $10^{5} \mathrm{~s}^{-1}$ - well below the quenching rate and conditions in which non-equilibrium effects become relevant. From these results it can be concluded that non-equilibrium chemistry effects are secondary for the here investigated rocket-injector configuration. Although effects of wall-heat losses have not been considered in this analysis, this conclusion will also extend to non-adiabatic flows since convective heat-losses evolve on time-scales that are long compared to chemical time-scales. 


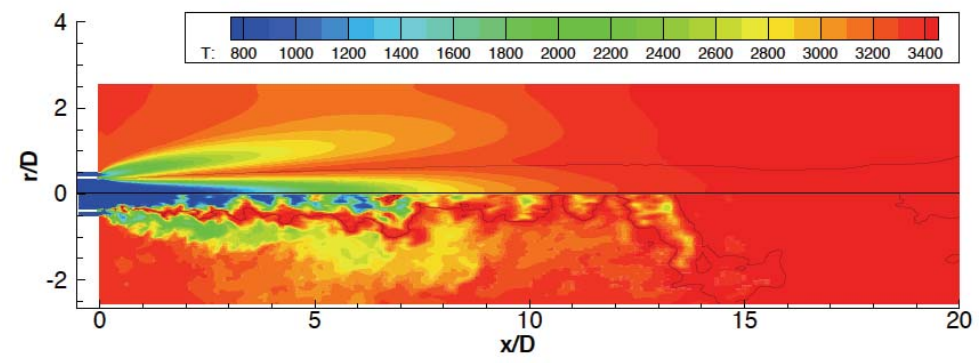

(a) Equilibrium FPV-model, FPV $\{$ Equ, Adiab $\}$.

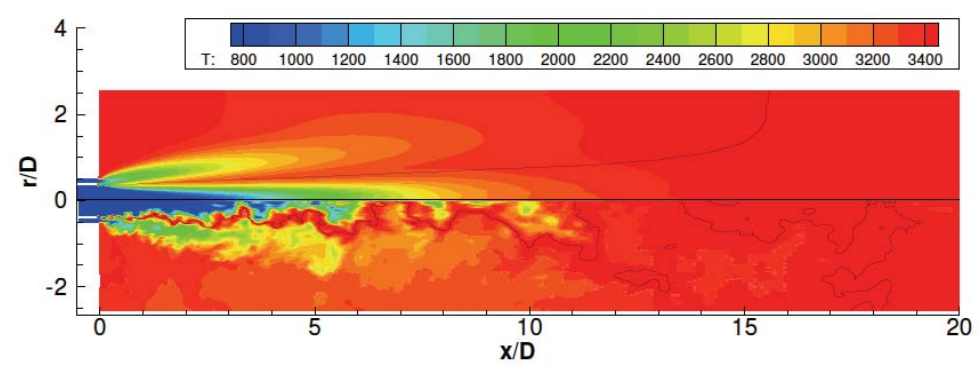

(b) Adiabatic FPV-model, FPV $\{$ Adiab $\}$.

Figure 7. Comparison of mean (top) and instantaneous (bottom) temperature field obtained from (a) the equilibrium combustion model and (b) non-equilibrium model. The solid line indicates the stoichiometric mixture fraction isocontour with $Z_{\text {st }}=0.2273$.

\section{B. Non-Adiabatic Results}

To assess effects of wall-heat transfer on the flow-field structure, simulations with the non-adiabatic FPVmodel are conducted. Statistical flow-field results and comparisons against results from the adiabatic models are illustrated in Figs. 10-12. The mean temperature in the combustion chamber computed with the nonadiabatic (top) and adiabatic (bottom) model are shown in Fig. 10. From this direct comparison several observations can be made. First, the boundary layer is mainly apparent in the recirculation region and the combustor end wall. The thickness of the boundary layer increases with increasing down-stream distance, which is also evident from the radial profiles presented in Fig. 11. Second, considerable effects of the wallheat loss on the flame/flow-field structure can be observed in the region up to eight diameters downstream of the combustor end wall. In this region the flame temperature decreases by as much as $500 \mathrm{~K}$ throughout the combustion chamber. With increasing downstream distance the temperature inside the combustor core region equilibrates, and heat-loss effects become increasingly confined to the combustor-wall region (see for instance temperature profiles for $x / D=20.03$ in Fig. 11).

The hydroxyl-radical mass fraction also shows a pronounced sensitivity to the temperature field. Specifically, it can be seen from Fig. 12 that for $x / D<5$ the mean OH-mass fraction is almost completely diminished outside the flame-zone, and $\mathrm{OH}$ mass fraction at $x / D=20$ is reduced by approximately 25 percent compared to the adiabatic predictions.

Overall, considerable differences can be observer for the LES-computations in which heat-loss effects are considered. These effects are most prominent in the recirculation region where the residence times are large. Although the boundary layer is not fully resolved and radiative heat-losses have not been incorporated in this calculation, it is anticipated that the consideration of these effects will result in further increase in heat-losses and increased variation in the combustor flow field. 

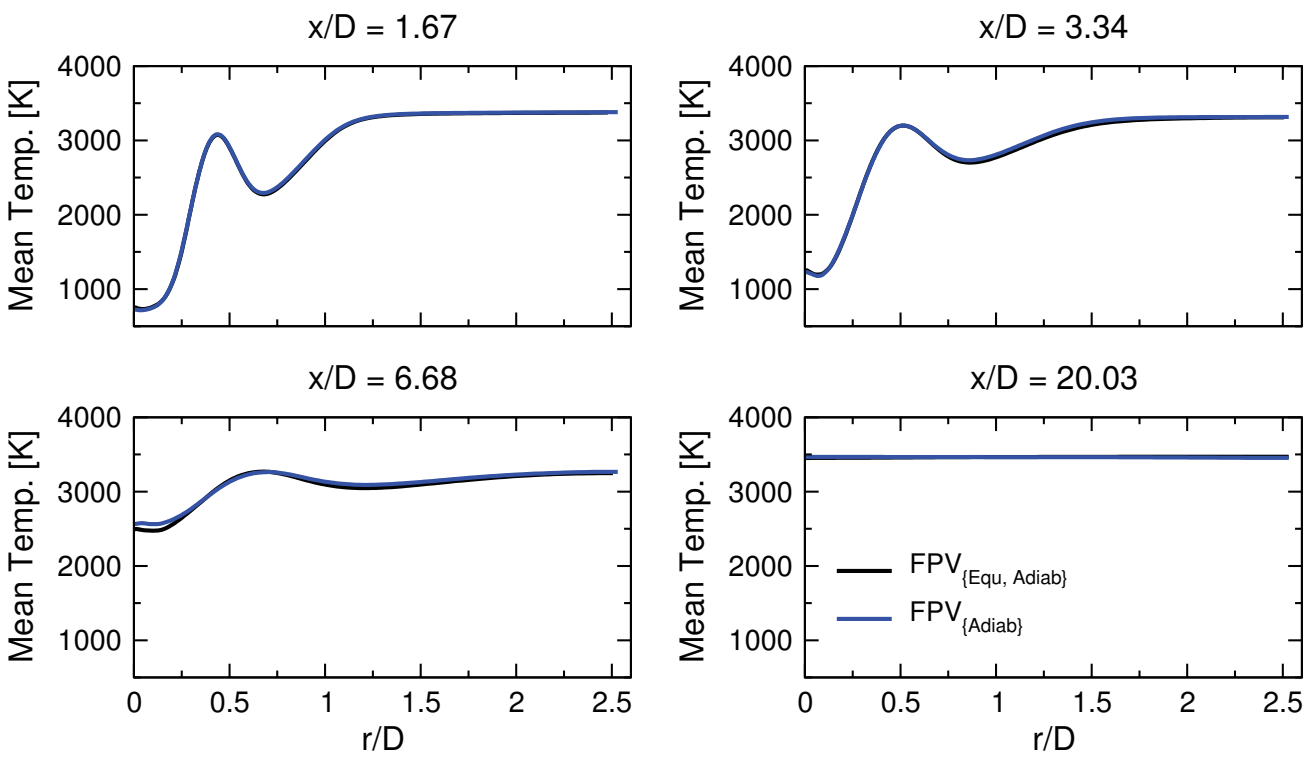

Figure 8. Comparison of radial profiles of mean temperature computed at four different downstream locations in the rocket-combustor.

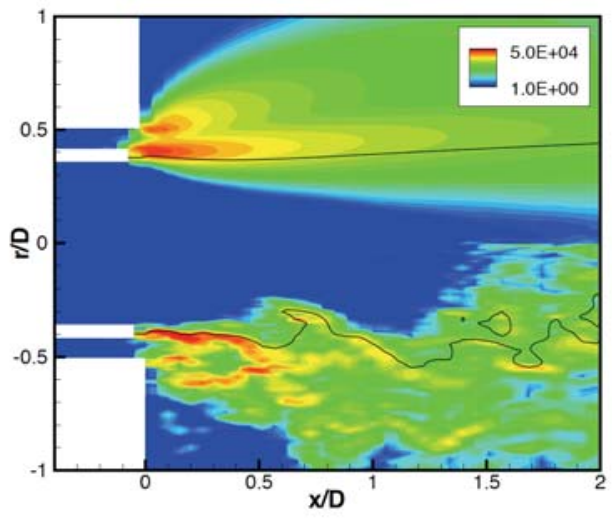

Figure 9. Mean (top) and instantaneous (bottom) scalar dissipation rate; solid line shows the isocontour of stoichiometric mixture fraction.

\section{Conclusions}

A LES combustion model for predicting wall-heat transfer in confined combustors and rocket engines has been developed. This model extends an adiabatic flamelet-formulation by including a source term to account for convective heat-loss effects. The time scale that is associated with the heat-loss is modeled through a Nusselt-number relation. Using the formulation, unsteady non-adiabatic flamelet equations are solved. The thermochemical composition of these unsteady flamelets is then parameterized in terms of mixture fraction, temperature, and scalar dissipation rate.

Compared to previously developed models that utilize a defect-enthalpy, the present non-adiabatic formulation offers the advantage that the model does not depend on the explicit specification of an enthalpy function. Furthermore, this model is able to account for flame-quenching due to rapid and localized heat-losses. A presumed PDF-closure model is employed in order to account for the turbulence/chemistry interaction in this high-Reynolds number combustion environment.

The non-adiabatic combustion model was applied to LES of a uni-element rocket injector, and comparisons with adiabatic simulations were performed in order to assess effects of non-equilibrium chemistry and wall-heat losses on the flow-field structure. Simulation results suggest that for the present single-injector 


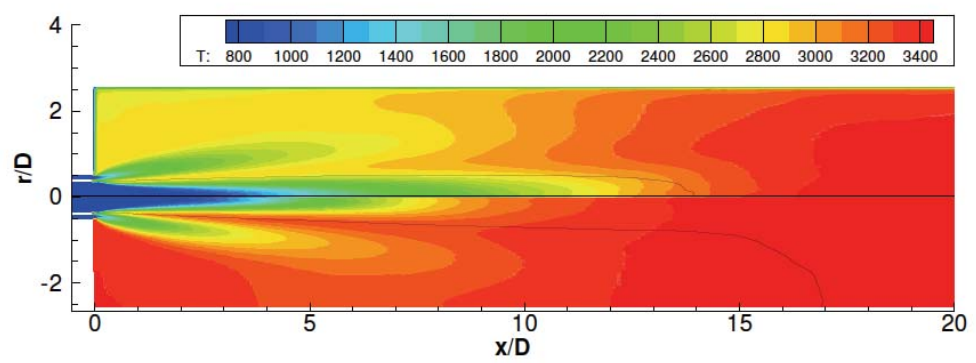

Figure 10. Comparison of the mean temperature field obtained from (top) non-adiabatic FPV $\{$ Non-Adiab $\}$ model and (bottom) adiabatic PPV $_{\{\text {Equ, Adiab }}$-formulation.
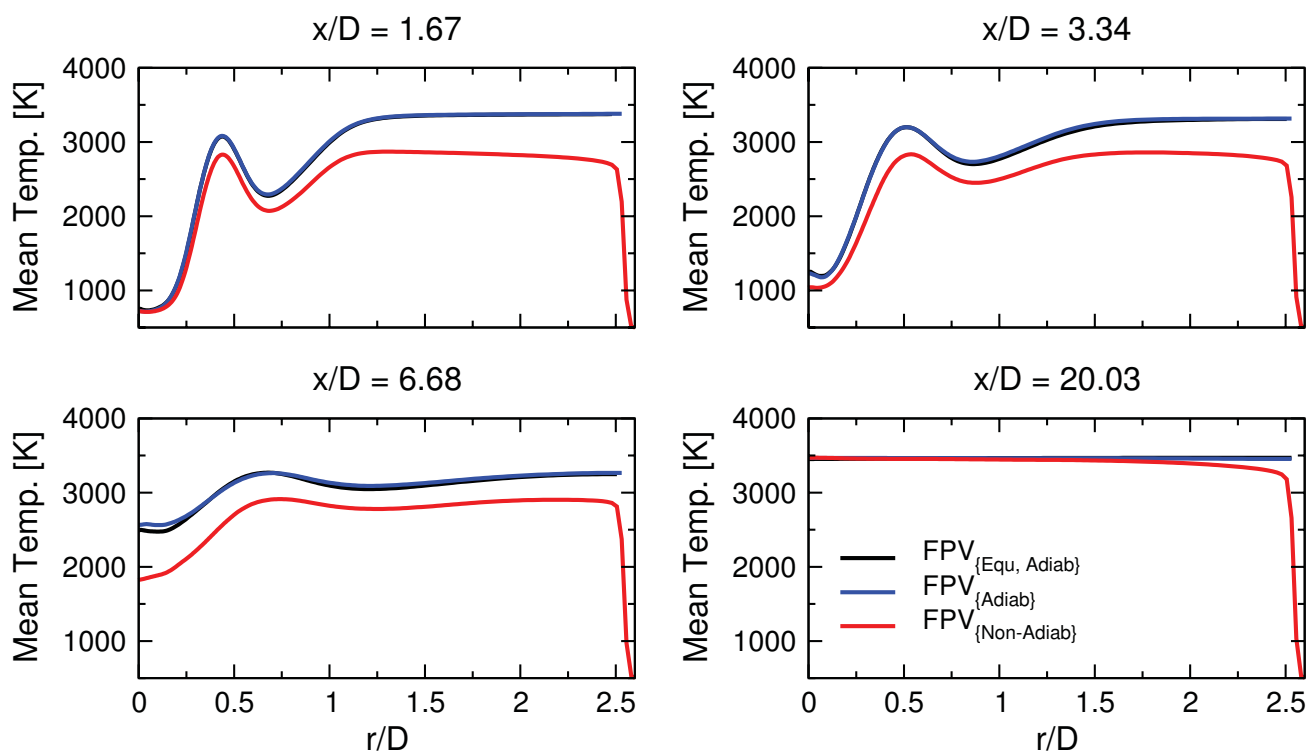

Figure 11. Comparison of radial profiles of mean temperature computed at four different downstream locations in the rocket-combustor.

configuration strain-rate effects are not significant so that the species distribution can be represented by its equilibrium composition.

Model predictions obtained with the non-adiabatic combustion model show a pronounced effect of wallheat losses on the temperature and species composition. These effects are not only confined to the near-wall region, but are also evident in the combustor core region and recirculation region that are characterized by long residence times. In particular, these heat-loss effects can result in temperature reductions by as much as $500 \mathrm{~K}$ in the injector-near region, and reductions of OH-mass fractions by $25 \%$ at the combustor exit. In the current simulation, the wall-region is not fully resolved and it is anticipated that an improved description of the near-wall region results in a stronger coupling between heat-loss effects and flow-field structure. This aspect is the topic of ongoing research.

\section{Acknowledgments}

Financial support through NASA is gratefully acknowledged. 

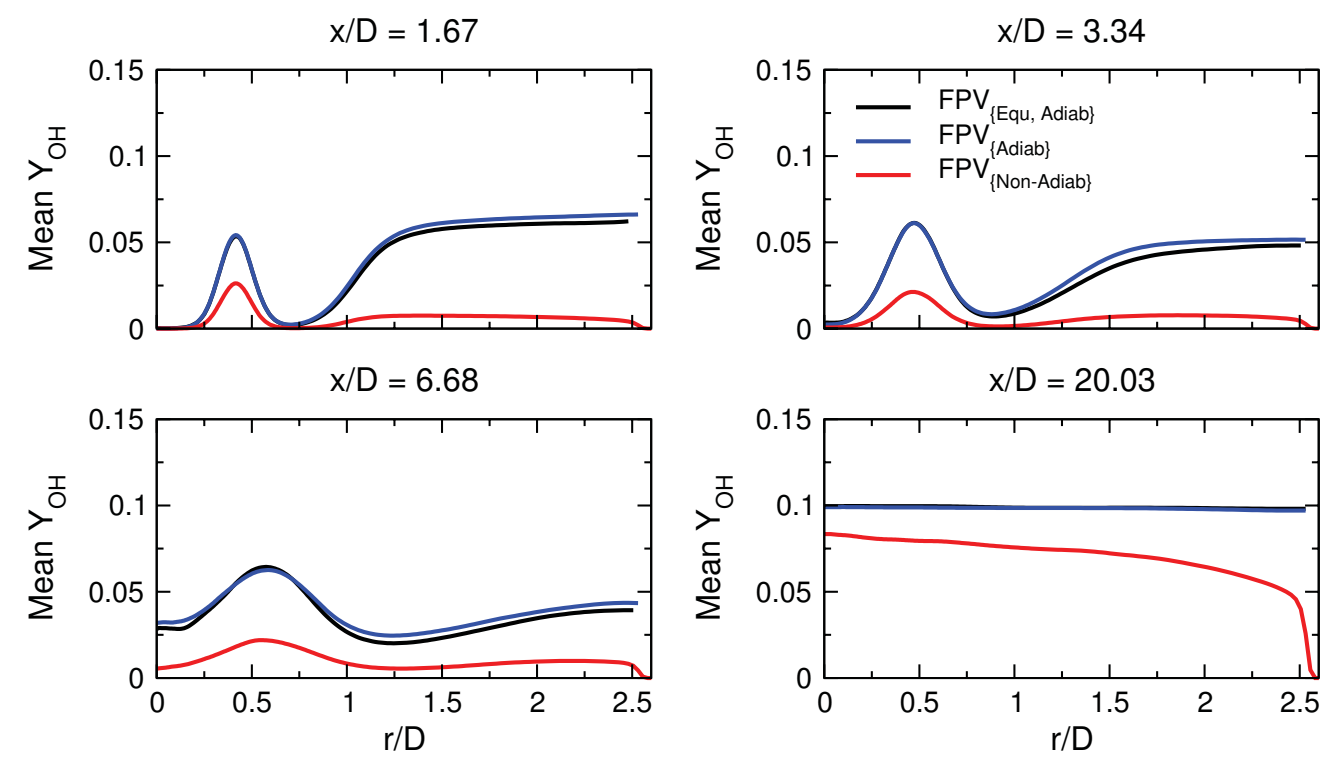

Figure 12. Comparison of radial profiles of mean OH-mass fraction computed at four different downstream location in the rocket-combustor.

\section{References}

${ }^{1}$ Monk, J. C. and Goetz, O. K., "Combustion device failures during space shuttle main engine development," 5th International Symposium on Liquid Space Propulsion Long Life Combustion Devices Technology, Chattanooga, TN, 2003.

${ }^{2}$ Tucker, P. K., Menon, S., Merkle, C. L., Oefelein, J. C., and Yang, V., "Validation of High-Fidelity CFD Simulations for Rocket Injector Design," AIAA Paper 2008-5226, 2008.

${ }^{3}$ Pal, S., Marshall, W., Woodward, R., and Santoro, R., "Wall heat flux measurements for a uni-element GO2/GH2 shear coaxial injector," 2006, Third International Workshop on Rocket Combustion Modeling, Paris, France, March, 13-15.

${ }^{4}$ Sozer, E., Hassan, E. A., Yun, S., Thakur, S., Wright, J., Ihme, M., and Shyy, W., "Turbulence-Chemistry Interaction and Heat Transfer Modeling of $\mathrm{H}_{2} / \mathrm{O}_{2}$ Gaseous Injector Flows," AIAA Paper 2010-1525, 2010.

${ }^{5}$ Sozer, E., Modeling of gaseous reacting flow and thermal environment of liquid rocket injectors, Ph.D. thesis, University of Michigan, 2010.

${ }^{6}$ Bray, K. N. C. and Peters, N., "Laminar flamelets in turbulent flames," Turbulent Reacting Flows, edited by P. A. Libby and F. A. Williams, Academic Press, London, 1994, pp. 63-113.

${ }^{7}$ Marracino, B. and Lentini, D., "Radiation modelling in non-luminous nonpremixed turbulent flames," Combust. Sci. Tech., Vol. 128, No. 1-6, 1997, pp. 23-48.

${ }^{8}$ Hossain, M., Jones, J. C., and Malalasekera, W., "Modelling of a bluff-body nonpremixed flame using a coupled radiation/flamelet combustion model," Flow, Turb. Combust., Vol. 67, No. 3, 2001, pp. 217-234.

${ }^{9}$ Navarro-Martinez, S., Kronenburg, A., and di Mare, F., "Conditional Moment Closure for Large Eddy Simulation," Flow, Turb. Combust., Vol. 75, No. 1-4, 2005, pp. 245-274.

${ }^{10}$ Shunn, L., Large-Eddy Simulation of Combustion Systems with Convective Heat-Loss, Ph.D. thesis, Stanford University, 2009.

${ }^{11}$ Barlow, R. S., 1996, Web site for the International Workshop on Measurement and Computation of Turbulent Nonpremixed Flames (TNF), http://www.ca.sandia.gov/TNF/.

${ }^{12}$ Kuznetsov, V. R. and Sabel'nikov, V. A., Turbulence and Combustion, Hemishpere, New York, 1990.

${ }^{13}$ Barlow, R. S., Karpetis, A. N., Frank, J. H., and Chen, J.-Y., "Scalar Profiles and NO Formation in Laminar OpposedFlow Partially Premixed Methane/Air Flames," Combust. Flame, Vol. 127, No. 3, 2001, pp. 2102-2118.

${ }^{14}$ Peters, N., "Local quenching due to flame stretch and non-premixed turbulent combustion," Combust. Sci. Tech., Vol. 30, 1983, pp. 1-17.

${ }^{15}$ Peters, N., "Laminar diffusion flamelet models in non-premixed turbulent combustion," Prog. Energy Combust. Sci., Vol. 10, No. 3, 1984, pp. 319-339.

${ }^{16}$ Williams, F. A., "Descriptions of Nonpremixed Turbulent Combustion," AIAA Paper 2006-1505, 2006.

${ }^{17}$ Peters, N., Turbulent Combustion, Cambridge University Press, Cambridge, 2000.

${ }^{18}$ Gnielinski, V., "Neue Gleichungen für den Wärme- und den Stoffübergang in turbulent durchströmten Rohren und Kanälen," Forsch. Ing.-Wes., Vol. 41, No. 1, 1975, pp. 8-16.

${ }^{19}$ Ihme, M. and Pitsch, H., "Modeling of Radiation and NO Formation in Turbulent Non-premixed Flames Using a Flamelet/Progress Variable Formulation," Phys. Fluids, Vol. 20, 2008, pp. 055110.

${ }^{20}$ Germano, M., Piomelli, U., Moin, P., and Cabot, W. H., "A dynamic subgrid-scale eddy viscosity model," Phys. Fluids A, Vol. 3, No. 7, 1991, pp. 1760-1765. 
${ }^{21}$ Lilly, D. K., "A proposed modification of the Germano subgrid-scale closure method," Phys. Fluids A, Vol. 4, No. 3, 1992, pp. 633-635.

${ }^{22}$ Pierce, C. D. and Moin, P., "Progress-variable approach for large-eddy simulation of non-premixed turbulent combustion," J. Fluid Mech., Vol. 504, 2004, pp. 73-97.

${ }^{23}$ Ihme, M., Cha, C. M., and Pitsch, H., "Prediction of local extinction and re-ignition effects in non-premixed turbulent combustion using a flamelet/progress variable approach," Proc. Combust. Inst., Vol. 30, 2005, pp. 793-800.

${ }^{24}$ Pitsch, H., "FlameMaster v3.1: A C++ computer program for 0D combustion and 1D laminar flame calculations," 1998, available from http://www.stanford.edu/group/pitsch/.

${ }^{25}$ O'Conaire, M., Curran, H. J., Simmie, J. M., Pitz, W. J., and Westbrook, C. K., "A Comprehensive Modeling Study of Hydrogen Oxidation," Int. J. Chem. Kinet., Vol. 36, 2004, pp. 603-622. 